\title{
ON THE DIRECT-PRODUCT OF OPERATOR ALGEBRAS II
}

\section{TAKASI TURUMARU}

(Received September 2, 1952)

1. Introduction. R. Schatten-J. von Neumann [4] introduced the idea of direct-product of Banach spaces, and the author modified this considerations to $C *$-algebras in the previous paper [7], and defined the directproduct of $C^{*}$-algebras.

Let $A_{1}$ and $A_{\text {. }}$ be any $C^{*}$-algebras with unit, and following R. Schatten-J. von Neumann. construct $A_{1} \times A_{2}$ as the set of all expressions $\sum x_{i} \times y_{i}$ with the equivalence relation $\cong$, as $A_{1}, A_{2}$ to be Banach spaces; and finally define the multiplication, involution and norm of expressions as follows:

product : $\quad\left(\sum_{i=1}^{n} x_{i} \times y_{i}\right)\left(\sum_{j=1}^{m} s_{i} \times t_{j}\right)=\sum_{i=1}^{n} \sum_{j=1}^{m} x_{i} s_{j} \times y_{i} t_{j}$

involution :

$$
\left(\sum_{i=1}^{n} x, \times y_{i}\right)^{*}=\sum_{i=1}^{n} x_{i}^{*} \times y_{i}^{*} \text {, }
$$

norm : $\quad \alpha\left(\sum_{i=1}^{n} x_{i} \times y_{i}\right)=\sup \left[\Phi\left(\left(\sum_{i=1}^{n} x_{i} \times y_{i}\right)\left(\sum_{i=1}^{n} x_{i} \times y_{i}\right)^{*}\right)^{1 / 2}: \Phi \in \varsigma\right]$,

where $\odot$ denotes the set of positive type functional $\Phi$ 's such that

$$
\Phi\left(\sum_{j=1}^{m} s_{i} \times t_{i}\right)=\frac{\varphi \times \psi\left(\left(\sum_{i=1}^{n} x_{i} \times y_{i}\right)\left(\sum_{i=1}^{m} s_{j} \times t_{j}\right)\left(\sum_{i=1}^{n} x_{i} \times y_{i}\right)^{*}\right)}{\psi \times \psi\left(\left(\sum_{i=1}^{n} x_{i} \times y_{i}\right)\left(\sum x_{i} \times y_{i}\right)^{*}\right)}
$$

$\psi$ and $\psi$ are pure states on $A_{1}$ and $A_{2}$ respectively, and $\sum_{i=1} x_{i} \times y_{i}$ is an arbitrary element of $A_{1} \times A_{2}$; then $\alpha$ becomes a cross-norm on $A_{1} \times A_{2}$ and $A_{1} \times A_{2}$, is a non.complete $C^{*}$-algebra [7].

Now, let $A_{1}$ and $A_{2}$ be $C^{*}$-algebras on the Hilbert spaces $H_{1}$ and $H_{2}$ respectively. Then by $[3,4], \sum_{i=1}^{n} x_{i} \times y_{i}$ can be considered as bounded operator on $H=H_{1} \times{ }_{\sigma} H_{2}$. In this paper, we consider the relation between $C^{*}$-algebra generated by $\sum_{i=1}^{n_{0}} x_{i} \times y_{i}$ as operator on $H$ with operator bound as norm, and direct-product $A_{1} \times_{a} A_{2}(\S 2)$; and we give more detailed discussions in the case where $A_{i}$ are $C^{*}$-algebras of completely continuous operators on $H_{\varepsilon}(\S 3)$; and finally in $\S 4$ we prove some algebraic properties of $A_{1} \times{ }_{a} A_{2}$. 
2. Relation between the direct-product as operators and as algebras.1) Suppose that $H_{1}$ and $H_{2}$ are Hilbert spaces. Then, F. J. Murray-J. von Neumann's construction [3] of direct-product gives us $H=H_{1} \times{ }_{\sigma} H_{2}$ as Hilbert space. If $x$ and $y$ are operators on $H_{1}$ and $H_{2}$ respectively, then

$$
\left(\sum_{i=1}^{n} \xi_{i} \times \eta_{i}\right)(x \times y)=\sum_{i=1}^{n} \xi_{i} x \times \eta_{i} y
$$

gives a linear operator $x \times y$ on $H$, and the operator bound of $x \times y$ on $H$ satisfies the cross-property of Schatten [4]: $\|x \times y\|=\|x\| \cdot\|y\| \|^{2}$.

If $A_{1}$ and $A_{2}$ are $C^{*}$-algebras on $H_{1}$ and $H_{2}$ respectively, then the set $\left\{x \times y: x \in A_{1}, y \in A_{2}\right\}$

generates a $C^{*}$-algebra $A$.

On the other hand, we can consider the direct-product $A_{1} \times{ }_{\alpha} A_{z}$ as in [7], in this case, we define the norm $\left.\alpha()^{\circ}\right)$ as follows :

$$
\alpha\left(\sum_{i=1}^{n} x_{i} \times y_{i}\right)=\sup \left[\Phi\left(\left(\sum_{i=1}^{n} x_{i} \times y_{i}\right)\left(\sum_{i=1}^{n} x_{i} \times y_{i}\right)^{*}\right)^{1 / 2}: \Phi \in \mathcal{S}^{\prime}\right],
$$

where $\mathcal{S}^{\prime}$ denotes the set of positive type functional $\Phi$ 's on $A_{1} \times A_{2}$ such that $\Phi=\sum_{i, j=1}^{n} \varphi_{i j} \times \psi_{i j}, \Phi(1 \times 1)=1$, and $\varphi_{i j}(x), \psi_{i, j}(y)$ have the form $\phi_{i j}(x)=\left\langle\xi_{i} x, \xi_{j}\right\rangle$, and $\psi_{i j}(y)=\left\langle\eta_{i} y, \eta_{j}\right\rangle$

respectively where $\xi_{i} \in H_{1}, \eta_{j} \in H_{2}$ and $\mid \sum_{i=1}^{n} \xi_{i} \times \eta_{j} \|=1$. Then the following theorem holds :

THEOREM 1. A is isometrically isomorphic to the direct-product $A_{1} \times_{\alpha} A_{2}$.

LEMMA 1. If the expression $\sum_{i=1}^{n} x_{i} \times y_{i}$ is equivalent to $0 \times 0$ as element of direct product $A_{1} \times_{\text {a }} A_{2}^{\prime}$ of algebras, then it is zero operator on $H$.

Proof. By the definition of the norm in $A_{1} \times{ }_{\alpha} A_{2}$,

$$
\begin{aligned}
0 & =\sum_{i, j=1}^{n}<\xi x_{i} x_{j}^{*}, \xi>\left\langle\eta y_{i} y_{j}^{*}, \eta\right\rangle \\
& =\sum_{i, j=1}^{n}<\xi x_{i}, \xi x_{j}><\eta y_{i}, \eta y_{j}> \\
& =<\sum_{i=1}^{n} \xi x_{i} \times \eta y_{i}, \sum_{j=1}^{n} \xi x_{j} \times \eta y_{j}>
\end{aligned}
$$

for any elements $\xi \in H_{1}, \eta \in H_{2},\|\xi\|=\|\eta\|=1$. Then, $\sum_{i=1}^{n} \xi x_{i} \times \eta y_{i}=0$ for any $\xi \in H_{1}, \quad \eta \in H_{2}$, so $\sum_{i=1}^{n} x_{i} \times y_{i}=0$ as operator on $H$.

1) The author expresses his hearty thanks for many discussions of Prof. M. Nakamura; he has pointed out the incompleteness of author's original proof of present and next sections.

2) III $\mid$ II denotes the operator bound. 
LEMMA 2. The converse statement of Lemma 1 holds.

PROOF. Let $\sum_{i=1}^{n ?} x_{i} \times y_{i}=0$ as operator on $H$. First we rremark that we can assume, without loss of generality, the linear independency of $\left\{x_{i}\right\}$. Indeed, if $x_{1}=\sum_{i=2}^{n} a_{i} x_{i}$ holds, then for any $\xi \times \eta \in H$,

$$
\begin{aligned}
(\xi \times \eta) \sum_{i=1}^{n} x_{i} \times y_{i} & =(\xi \times \eta)\left(\left(\sum_{i=2}^{n} a_{i} x_{i}\right) \times y_{1}+\sum_{i=2}^{n} x_{i} \times y_{i}\right) \\
& \cong \sum_{i=2}^{n} a_{i} \xi x_{i} \times \eta y_{1}+\sum_{i=2}^{n} \xi x_{i} \times \eta y_{i} \\
& \cong \sum_{i=2}^{n} \xi x_{i} \times \eta\left(a_{i} y_{1}\right)+\sum_{i=2}^{n} \xi x_{i} \times \eta y_{i} \\
& \cong(\xi \times \eta)\left(\sum_{i=2}^{n} x_{i} \times\left(a_{i} y_{1}+y_{i}\right)\right)
\end{aligned}
$$

so $\sum_{i=1}^{n} x_{i} \times y_{i} \cong \sum_{i=2}^{n} x_{i} \times\left(a_{i} y_{1}+y_{i}\right)$.

Now, assume that $\sum_{i=1}^{n} x_{i} \times y_{i}=0$ as operator on $H$, and $\left\{x_{i}\right\}$ are linearly independent, then for any $\xi, \xi^{\prime} \in H_{1}$ and $\eta, \eta^{\prime} \in H_{2}$

$$
\begin{aligned}
0 & =<(\xi \times \eta) \sum_{i=1}^{n} x_{i} \times y_{i}, \quad \xi^{\prime} \times \eta^{\prime}> \\
& =<\sum_{i=1}^{n} \xi x_{i} \times \eta y_{i}, \quad \xi^{\prime} \times \eta^{\prime}> \\
& =\sum_{i=1}^{n}<\xi x_{i}, \quad \xi^{\prime}><\eta y_{i}, \quad \eta^{\prime}> \\
& =\sum_{i=1}^{n}<<\eta y_{i}, \quad \eta^{\prime}>\xi x_{i}, \quad \xi^{\prime}> \\
& =<\sum_{i=1}^{n}<\eta y_{i}, \quad \eta^{\prime}>\xi x_{i}, \quad \xi^{\prime}>.
\end{aligned}
$$

Since $\xi^{\prime}$ is any element of $H_{1}, \Sigma<\eta y_{i}, \eta^{\prime}>\xi x_{i}=\xi\left(\Sigma<\eta y_{i}, \quad \eta>^{\prime} x_{i}\right)=0$, and furthermore, by the arbitrariness of $\xi \in H_{1}, \quad \Sigma<\eta y_{i}, \quad \eta^{\prime}>x_{i}=0$ as operator on $H_{1}$. While $\left\{x_{i}\right\}$ are linearly independent, so $\left\langle\eta y_{i}, \eta^{\prime}>=0\right.$ for $i=1,2, \cdots, n$. Again by the arbitrariness of $\eta$, and $\eta^{\prime}, y_{i}=0, i=1,2$, $\ldots, n$ as operators on $H_{2}, \quad$ so finally $\sum_{i=1}^{n} x_{i} \times y_{i} \cong 0 \times 0$ as element of direct-product. 
LeMMA 3.

$$
\alpha\left(\sum_{i=1}^{n} x_{i} \times y_{i}\right)=\left\|\sum_{i=1}^{n} x_{i} \times y_{i}\right\|
$$

Proof.

$$
\begin{aligned}
& \left\|\sum_{i=1}^{n} x_{i} \times y_{i}\right\|_{i}^{\prime 2}=\sup \left[\left|\left(\sum_{i=1}^{n^{\prime}} \xi_{j} \times \eta_{j}\right)\left(\sum_{i=1}^{n} x_{i} \times y_{i}\right)^{2}: \| \sum_{j=1}^{n^{\prime}} \xi_{i} \times \eta_{j}\right|=1\right] \\
& =\sup \left[\left\|\sum_{i, j} \xi_{j} x_{i} \times \eta_{j} y_{i}\right\|^{2}:\left\|\sum_{j} \xi_{j} \times \eta_{i}\right\|=1\right] \\
& =\sup \left[\sum_{i, j, k, m}<\xi_{j} x_{i}, \xi_{k} x_{m}><\eta_{i} y_{i}, \eta_{k} y_{m}>:\left\|\sum_{j} \xi_{i} \times \eta_{3}\right\|=1\right] \\
& =\sup \left[\sum_{i, j, k, m} \phi_{j i n}\left(x_{i} x_{i \prime}^{*}\right) \psi_{i k:}\left(y_{i} y_{m}^{*}\right):\left\|\sum_{j} \xi_{j} \times \eta_{j}\right\|=1\right] \text {, } \\
& \text { where } \phi_{j k}(x)=\left\langle\xi_{j} x, \xi_{k}\right\rangle \text { and } \psi_{j k}(y)=\left\langle\eta_{j} y, \quad \eta_{k}\right\rangle \\
& =\sup \left[\Phi\left(\left(\sum_{i=1}^{n} x_{i} \times y_{i}\right)\left(\sum_{j=1}^{n} x_{j}^{*} \times y_{j}^{*}\right)\right): \Phi \in \Theta^{\prime}\right] \\
& =\alpha\left(\sum_{i=1}^{n} x_{i} \times y_{i}\right)^{2} .
\end{aligned}
$$

Proof of Theorem 1. By Lemmas 1 and 2, $A$ is algebraically isomorphic to $A_{1} \times_{\alpha} A_{2}$, and by Lemma 3 , this isomorphism is also isometric. This completes the proof.

3. Direct-product of completely, continuous operators. Our principal aim of this section is to show

THEOREM 2. If $A_{1}$ and $A_{2}$ are $C^{k}$-algebras of completely continuous operators on $H_{1}$ and $H_{2}$ respectively, then $A=A_{1} \times_{a} A_{2}$ is also a $C^{*}$-algebra of completely continuous operators on $H=H_{1} \times{ }_{\sigma} H_{\text {.. }}$.

To prove the statement, we shall begin by proving

LEMMA 4. For any $\xi_{1}, \xi_{2} \in H_{1}$ and $\eta_{1}, \eta_{2} \in H_{2}$,

$$
\left(\xi_{1} \times \eta_{1}\right) \times\left(\xi_{2} \times \eta_{2}\right)=\left(\xi_{1} \times \xi_{2}\right) \times\left(\eta_{1} \times \eta_{2}\right)
$$

where $\zeta\left(\zeta_{1} \times \zeta_{2}\right)=\left\langle\zeta, \zeta_{2}>\zeta_{1} f(r) \zeta, \zeta_{1}, \zeta_{z} \in H\right.$.

Proof. Let $\xi \in H_{1}$ and $\eta \in H_{2}$, we have, forl $\zeta=\xi \times \eta$,

$$
\begin{aligned}
& (\xi \times \eta)\left(\left(\xi_{1} \times \eta_{1}\right) \times\left(\xi_{2} \times \eta_{2}\right)\right) \\
& =<\xi \times \eta, \xi_{2} \times \eta_{2}>\xi_{1} \times \eta_{1} \\
& =<\xi, \xi_{2}><\eta, \eta_{2}>\xi_{2} \times \eta_{2} \\
& =\xi\left(\xi_{1} \times \xi_{2}\right) \times \eta\left(\eta_{1} \times \eta_{2}\right)
\end{aligned}
$$




$$
=(\xi \times \eta)\left[\left(\xi_{1} \times \xi_{2}\right) \times\left(\eta_{1} \times \eta_{2}\right)\right] .
$$

To prove the the rre $\mathrm{n}$, it is sufficient to show that each $x \times y$ is completely continuous, since $A_{1} \times{ }_{\alpha} A_{2}$ is generated by such $x \times y$ 's. If $x=\sum_{=1}^{\infty} \xi_{i} \times \xi_{i}$ and $y=\sum_{j=1}^{\infty} \eta_{j} \times \eta_{j}^{\prime}$, are the canonical form of J.Dixmier [1], then it is also sufficient to show for

$$
x=\sum_{i=1}^{n} \xi_{i} \times \xi_{i}^{\prime} \text { and } y=\sum_{j=1}^{m} \eta_{j} \times \eta_{j}^{\prime}
$$

since such ${ }^{7} x \times y$ are dense in $A_{1} \times{ }_{a} A_{2}$. Therefore by Lemma 4 ,

$$
\begin{aligned}
x \times y & =\left(\sum_{i=1}^{n} \xi_{i} \times \xi^{\prime}\right) \times\left(\sum_{j=1}^{m} \eta_{j} \times \eta_{j}^{\prime}\right) \\
& =\sum_{i=1}^{n} \sum_{j=1}^{m}\left(\xi_{i} \times \eta_{j}\right) \times\left(\xi_{i}^{\prime} \times \eta^{\prime}\right)
\end{aligned}
$$

shows that $x \times y$ is a completely continuous operator on $H_{1} \times_{\sigma} H_{2}$. This proves Theorem 2 .

THEOREM 3. If $A_{i}$ of the previous theorem are the algebra $C\left(H_{i}\right)$ of all completely continuous operators on $H_{i}$, then $A$ is the algebra $C(H)$ of all completely conlinuous operators on $H$ : i.e.,

$$
C\left(H_{1}\right) \times_{\alpha} C\left(H_{2}\right)=C\left(H_{1} \times{ }_{\sigma} H_{1}\right) .
$$

Proof. By the previous theorem, it is sufficient to show that $A$ contains all one-dimensional projections of $H$. This is proved when $\left(\sum_{i=1}^{\infty} \xi_{i} \times \eta_{i}\right)$ $\times\left(\sum_{i=1}^{\infty} \xi_{i} \times \eta_{1}\right)$ is contained in $A$, if $\sum_{i=1}^{\infty} \xi_{i} \times \eta_{i}$ exists and is of norm unity. Since, for each $n$,

$$
\frac{\sum_{i=1}^{n} \xi_{i} \times \eta_{i}}{\left\|\sum_{i=1}^{n} \xi_{i} \times \eta_{s}\right\|} \times \frac{\sum_{i=1}^{n} \xi_{i} \times \eta_{i}}{\left\|\sum_{i=1}^{n} \xi_{i} \times \eta_{i}\right\|}
$$

exists in $A$ and converges uniformly to $\left(\Sigma \xi_{i} \times \eta_{i}\right) \times\left(\Sigma \xi_{i} \times \eta_{i}\right)$, the latter is contained in $A$.

Following Corollary is an immediate consequence of our Theorem 3 and I. Kaplansky [2].

COROLlary. If two $C^{*}$-algebras of completely continuous operators are simple, then their direct-product is simple too.

REMARK. This corollary is not yet decided when the condition of "completely continuous operators" is replaced by "operators." 
4. Some algebraic properties of $A_{1} \times_{a} A_{2}$.

Theorem 4. If $A_{i}$ are $C^{*}$-algebra with unit, and if $A_{1} \times_{a} A_{2}$ is simple, in the sense of non-existence of proper two-sided closed ideals, then each $A_{\text {i }}$ is simple.

Proof. We prove this theorem by an indirect argument. If $A_{1}$ were not simple, then there exists a proper closed ideal $I_{1}$. Then $I_{1} \times_{a} A_{2}$ is a closed proper ideal in $A_{1} \times{ }_{a} A_{2}$, so by assumption, $I_{1} \times{ }_{a} A_{2}=A_{1} \times{ }_{a} A_{2}$. Then for every positive real number $\varepsilon$, there exists $\sum_{i=1}^{r} x_{i} \times y_{i} \in J_{1} \times A_{2}$ such that

$$
\alpha\left(\sum_{\imath=1}^{n} x_{\imath} \times y_{\imath}-1 \times 1\right)<\varepsilon .
$$

Then, by the definition of $\alpha(\cdot)[7]$,

$$
\left|\sum_{i=1}^{n} \varphi\left(x_{i}\right) \psi\left(y_{i}\right)-1\right|<\varepsilon
$$

for any pure states $\varphi$ and $\psi$ on $A_{1}$ and $A_{2}$ respectively.

On the other hand, by I.E. Segal's result [6], there exists a pure state $\varphi_{\mathrm{c}}$ of $A_{1}$ which vanishes on $I_{1}$. For a such state $\varphi_{0}$,

$$
\varphi_{0}\left(x_{i}\right)=0, i=1,2, \cdots \cdots \text {. }
$$

Then the above inequality implies the contradiction $:|1|<\varepsilon$.

TheOREM 5. If $A_{i}$ are $C^{*}$-algebras with unit, and if $A_{1} \times_{\alpha} A_{2}$ is factorial (that is, the center is a multiple of unit), then each $A_{i}$ is so.

Proof. If $x$ is in the center of $A_{1}, x \times 1$ is in the center of $A_{1} \times_{a} A_{2}$, sc by assumption $x \times 1=a(1 \times 1)=(a 1) \times 1$ for some scalar $a$. Thus $x=a 1$, this is desired.

TheOREM 6. If $A_{1}=I+J$ (direct sum), then

$$
A_{1} \times{ }_{a} A_{2}=I \times{ }_{a} A_{2}+J \times{ }_{a} A_{2} \text { (direct sum). }
$$

Proof. By assumption, if $x \in A_{1}$, there exists a unique expression

$$
x=x^{\prime}+x^{\prime \prime}, x^{\prime} \in I, \quad x^{\prime \prime} \in J \text {. }
$$

Then for any $y \in A_{2}$,

$$
x \times y=x^{\prime} \times y+x^{\prime \prime} \times y, \quad x^{\prime} \times y \in I \times{ }_{a} A_{2}, \quad x^{\prime \prime} \times y \in J \times{ }_{a} A_{2} .
$$

Thus $(I+J) \times_{a} A_{2} \leqq I \times{ }_{a} A_{2}+J \times_{a} A_{2}$ (right-hand side is not necessarily direct). On the other hand, since $I+J \supseteqq I, J$,

$$
(I+J) \times_{a} A_{2} \supseteq I \times{ }_{a} A_{2}, J \times_{a} A_{2},
$$

so

$$
(I+J) \times_{a} A_{2} \supseteqq I \times A_{2}+J \times_{a} A_{2} .
$$

Thus, to prove the theorem it is sufficient to show

$$
\left(I \times_{a} A_{2}\right) \cap\left(I \times_{a} A_{2}\right)=0 .
$$

Now if $x^{\prime} \in I, x^{\prime \prime} \in J$, then for any $y^{\prime}, y^{\prime \prime} \in A_{2}$,

$$
\left(x^{\prime} \times y^{\prime}\right)\left(x^{\prime \prime} \times y^{\prime \prime}\right)=\left(x^{\prime} x^{\prime \prime}\right) \times\left(y^{\prime} y^{\prime \prime}\right)=0 \times\left(y^{\prime} y^{\prime \prime}\right)=0 \text {. }
$$


Since $I \times{ }_{a} A_{2}$ and $J \times_{a} A_{2}$ are generated from

$$
\left\{x^{\prime} \times y^{\prime}: x^{\prime} \in I, y^{\prime} \in A_{2}\right\} \text { and }\left\{x^{\prime \prime} \times y^{\prime \prime}: x^{\prime \prime} \in J, y^{\prime \prime} \in A_{2}\right\}
$$

respectively, $\left(I \times{ }_{a} A_{2}\right)\left(J \times_{\alpha} A_{2}\right)=0$, by the above relation.

On the other hand, $I \times_{\alpha} A_{2}$ and $J \times_{\alpha} A_{2}$ are closed two-sided ideals in $C^{*}$-algebra $A_{1} \times{ }_{\alpha} A_{2}$, so of course self-adjoint, then if $u \in I \times{ }_{a} A_{2} \cap J \times{ }_{a} A_{2}$, $u u^{*} \in\left(I \times_{a} A_{2}\right)\left(J \times_{a} A_{2}\right)=0$, that is $u u^{*}=0, u=0$. This completes the proof.

\section{BIBLIOGRAPHY}

1. J. DIXMIER, Les fonctionnelles linéaires sur l'ensemble des opérateurs bornés d'un espace de Hilbert, Ann. of Math., 51(1950), pp. 387-408.

2. I. KAPLANSKY, Dual Rings, Ann. of Math., 49(1948), pp. 589-701.

3. F. J.MURRAY-J. von NeumanN, On rings of operators, Ann. of Math., 37 (1936), pp. 116-229.

4. R. SChatten, A theory of cross-space, Princeton (1950).

5. I. E. SEGAL, Irreducible representations of operator algebras, Bull. Amer. Math. Soc., 53(1947), pp. 73-88.

6. I. E. SEGAL, Two-sided ideals in operator algebras, Ann. of Math.,50(1949), pp. 856865.

7. T. TURUMARU, On the direct-product of operator algebra I, Tôhoku Math. Journ., 4(1953), pp. 242-251.

Mathematical. Institute, TôHoku University, Sendai. 